\title{
Update on the management of Dupuytren's contracture
}

This article was published in the following Dove Press journal:

Orthopedic Research and Reviews

27 August 2010

Number of times this article has been viewed

\author{
Linda $\mathrm{Vi}^{\prime}$ \\ David B O'Gorman² \\ Bing Siang Gan ${ }^{3}$ \\ 'Department of Physiology and \\ Pharmacology, ${ }^{2} \mathrm{Hand}$ and Upper Limb \\ Centre, Lawson Health Research \\ Institute, Departments of Surgery \\ and Biochemistry, ${ }^{3} \mathrm{Hand}$ and Upper \\ Limb Centre, Lawson Health Research \\ Institute, Departments of Surgery \\ and Medical Biophysics, University of \\ Western Ontario, London, Ontario, \\ Canada
}

\begin{abstract}
Dupuytren's disease (DD) is a pathological condition of the palmar fascia that is characterized by the formation of tight collagenous disease cords leading to permanent finger contractures. The disease is most prevalent in Caucasian men, and its incidence increases with age advancement. The most common complaint from patients having DD is the impairment of normal hand function. At present, the disease is incurable and the pathophysiology of DD is unknown. The most common treatment for DD is surgery; however, this treatment is associated with a high rate of recurrence. More recently, researchers have begun to explore the molecular basis of DD in the hopes of developing new, more effective treatment for DD. This review will summarize the history and clinical presentation of the disease, highlight current and emerging molecular treatments, and explore the implications of these advancements for future work.
\end{abstract}

Keywords: Dupuytren's disease, etiology, clinical presentation, treatment

\section{Introduction to management issues in the treatment of Dupuytren's disease}

Dupuytren's contracture (or Dupuytren's disease [DD]) is a pathological condition affecting the palmar fascia of the hand resulting in flexion deformities of the affected fingers. ${ }^{1}$ This disease can also affect the plantar fascia, a condition called Ledderhose disease or plantar fibromatosis. Plantar fibromatosis is immunohistologically similar in presentation to DD of the palmar fascia, but unlike DD, patients with plantar fibromatosis do not typically develop contractures of the affected plantar structure. ${ }^{2}$ The DD affecting the hands often results in limitations in dexterity and can have a significant impact on an individual's ability to engage in vocational and avocational activities. Patients also report safety concerns, specifically expressing fear that the fixed flexion of the finger will make it more susceptible to injuries. ${ }^{3}$ Finally, patients living with DD also express concerns about how their contracture will affect their social contact, chiefly their ability to give a proper handshake. ${ }^{3}$ For these reasons, correction of flexion deformities is desirable. Unfortunately, in the absence of a full understanding of the molecular pathology, treatment is now merely aimed at eliminating the contracted pathologic disease tissue, without addressing the underlying pathophysiology.

In terms of treatment, the most common approach continues to be the surgical resection of the disease cord followed by intensive postoperative physiotherapy. ${ }^{4,5}$ Because of the significant morbidity associated with surgery, less extensive treatment approaches have been tried such as the needle aponeurotomy or enzymatic digestion of the disease cords. However, these procedures are not curative and are associated 
with a high rate of recurrence. ${ }^{6}$ Recent research has begun to explore the molecular basis of disease progression and recurrence in the hopes of developing new, more effective treatments for the management of DD. The objective of this review will be to (1) summarize the history and clinical presentation of the disease, (2) highlight current and emerging conventional and molecular treatments, and (3) explore the implications of these advancements for future work and enhanced patient care.

\section{Prevalence and etiology}

The prevalence of DD is influenced by age, gender, and ethnicity. This disease is thought to be an inherited dominant condition with variable penetrance that affects males 7-15 times more often than females. ${ }^{7}$ Typically, disease onset emerges after the fourth or fifth decade of life in males, whereas the disease tends to develop later in life for females. ${ }^{7,8}$ Although primary DD is more prevalent in males, after surgery the probability of recurrence is approximately equal in both the sexes. ${ }^{7}$ Although uncommon, DD has been reported in children and infants..$^{9-12}$ The disease has been reported in infants as young as 6 months old, ${ }^{11}$ and to date, there have been only a few histologically confirmed cases of DD identified in the literature. ${ }^{12}$ Within this pediatric age group, histological confirmation is strongly recommended to rule out other possibilities of digit contractures or nodule formation or both, such as camptodactyly, arthrogryposis, congenital ulnar drift, and epithelioid sarcoma. ${ }^{9,12}$ Because of the relatively low incidence of DD in children, many clinicians believe that this is an entity separate from classical DD.

DD is common in certain Caucasian populations, including Scandinavia, Britain, and Australia, but is less prevalent in other regions, such as Southern Europe, South America, and Asia. ${ }^{13,14}$ Although uncommon in most of Asia, certain populations within this area, specifically Japan and Taiwan, have higher prevalence, comparable to the values reported in Northern European populations. ${ }^{15}$ The prevalence of DD has been reported to be as high as $39.5 \%$ in males who are older than 70 years and are of Northern European descent. ${ }^{16}$

In the year 1963, Hueston ${ }^{13}$ first described a DD diathesis, a term that describes factors that would strongly predict disease severity and disease recurrence after treatment. Patients of Northern European descent, who present a younger age of onset, and those who have a positive family history of DD have an increased probability of developing the condition, as well as having a more aggressive disease progression. Hueston $^{13}$ also suggested that patients who have bilateral hand involvement and ectopic lesions (DD beyond the palm) are more likely to have aggressive disease progression and increased risk of recurrence. The severity of a patient's disease presentation continues to be an important factor in the management of this disease.

In addition to genetic factors, a number of comorbidities and socioenvironmental factors have been identified as contributing to this condition. Studies have suggested that there is increased risk for DD in patients with certain underlying health conditions, such as diabetes mellitus ${ }^{17,18}$ and epilepsy. ${ }^{19}$ Lifestyle risk factors that have been associated with DD include smoking and alcoholism, or high alcohol consumption. ${ }^{20-22}$ Other factors, such as the contributions of manual labour, ${ }^{23}$ hand or wrist trauma, ${ }^{24}$ and a history of repetitive hand vibrations, ${ }^{25}$ have also been associated with the disease. The contributions of these factors remain controversial, however, and other studies have been unable to identify any correlation between, eg, trauma and DD initiation. ${ }^{23,26,27}$

\section{Clinical presentation}

DD often occurs bilaterally, but typically affects one hand more severely than the other. The ring and small fingers are most often involved, though the other fingers and thumb may also be affected. ${ }^{28}$ The disease is believed to progress from a nodule to a cord ${ }^{29}$ that, as it matures, contracts and induces permanent fixed flexion of the affected digit(s). ${ }^{30}$

The earliest signs of DD involve changes in the skin, such as the presence of skin pitting. ${ }^{31}$ Skin pits are caused by fullthickness palmar skin retraction into the subcutaneous tissue. There can also be changes to the dorsum of the hand with the presence of knuckle pads or Garrod nodes. ${ }^{32}$ However, these are more prevalent in patients with bilateral disease and those with ectopic disease, such as plantar fibromatosis and Peyronie disease. ${ }^{33,34}$ Hence, it has been suggested that skin pits are a more reliable early indicator of DD.

A nodule is a palpable soft-tissue mass adhered to the skin and underlying palmar fascia and is often used as a diagnostic feature of the disease. ${ }^{29}$ These masses are typically painless, but can result in pain at the time of disease onset or when associated with tenosynovitis caused by the restriction of the flexor tendons. ${ }^{35}$ There are two main types of nodules: the palmar nodules found adjacent to the distal palmar crease and the digital nodules, which are commonly located near the proximal interphalangeal (PIP) joint or at the base of the digits.

As the disease progresses, a cord begins to form as a protrusion from the nodule. These cords seem to follow normal palmar fascial structures that in their healthy states are 
designated as "bands". As the cord matures, it becomes more fibrotic and takes on a more prominent appearance, superficially resembling a tendon. ${ }^{36}$ The contracture of the mature cord results in the distinct flexed position of the digits.

The most common cord formed in the palm, the so-called precentral cord arising from the precentral band of the palmar fascia, typically results in a flexion deformity of the metacarpophalangeal (MCP) joint. In some cases, the precentral cord may bifurcate at the level of the distal palmar crease; however, the cord rarely displaces the neurovascular bundle. ${ }^{37}$ Other cords that affect the palm include the vertical and natatory cords (arising respectively from the vertical bands of Legueu and Juvara, and the natatory ligaments), although these are less common than the precentral cord. ${ }^{38}$ Since the palmar fascia is continuous with the digital fascia, contractures of the palm often enter the digits, where they can induce additional flexion deformities of the PIP and distal interphalangeal (DIP) joints.

The most frequently encountered digital cords are the central, lateral, and spiral cords. ${ }^{30}$ The central cord is an extension of the precentral cord that forms in the midline of the neurovascular bundles and attaches to the flexor tendon sheath. This cord can cause deformities in the MCP joint and the PIP joint, but it rarely displaces the neurovascular bundles. The lateral cord is a continuation of the bifurcated precentral cord and is considered the pathological state of the lateral digital sheath. Unlike the central cord, it attaches to the skin or to the tendon sheath near the Grayson's ligament. This cord usually results in deformities of the PIP joint, but can also affect the DIP joint. In some instances, these lateral cords can result in the displacement of the neurovascular bundle toward the midline, complicating the surgical approach. The spiral cord is also an extension of the precentral cord that starts off deep in the neurovascular bundle and distal to the MCP joint. In the digit, the cord advances lateral to the neurovascular bundle to involve the lateral digital sheath and then superficial to the neurovascular bundle to involve Grayson's ligament. In doing so, the cord wraps itself around the neurovascular bundle, which causes the superficial, proximal, and central displacement of the neurovascular bundle when finger contractures are produced (Figure 1). This displacement also puts the neurovascular bundle at risk of being severed during surgery.

\section{Pathophysiology}

Although the precise pathophysiology of DD is unclear, this disease is often classified as a fibroproliferative condition. The pathogenesis of DD has been divided into three stages: (1) proliferative, (2) involution, and (3) residual. ${ }^{36,39}$ The proliferative stage is characterized by nodule formation, resulting from fibroblast hyperproliferation, myofibroblast differentiation, and type I and type III collagen deposition. ${ }^{36,39-41}$ Eventually, this process gives rise to the disease cord structure. During the involutional stage of disease progression, myofibroblasts align along the lines of tension of the diseased tissue. These cells are thought to generate contractile forces that lead to shortening of the diseased tissue with subsequent further collagen deposition permanently shortening the cord and causing joint contractures. ${ }^{36,41}$ Finally, during the residual stage, there is a further increase in type I collagen production. ${ }^{36,41}$ The disease cord also becomes more acellular in this stage, with increased myofibroblasts apoptosis. $^{36,41,42}$

It is generally believed that myofibroblast-generated contractile forces are largely responsible for the digit contractures in DD. This theory is based on extensive data demonstrating a temporal correlation between the appearance of myofibroblasts and the onset of contraction. ${ }^{43-45}$ Although these cells are of fibroblastic lineage, they express a number of specific muscle-associated markers, including $\alpha$-smooth muscle actin (a predominant isoform of actin found in contractile cells called myofibroblasts) and calponin, but lack other important smooth muscle markers, such as myosin. ${ }^{46}$ These myofibroblasts form extensive contacts to the extracellular matrix (ECM) at fibronectin-rich sites termed fibronexus. ${ }^{47,48}$ The disease cord tissue expresses high levels of fibronectin, and immunohistological analysis suggests that myofibroblasts within the disease cord interact with $\alpha_{5} \beta_{1}$ integrin-fibronectin complexes. ${ }^{49-51}$

On a biochemical level, DD resembles an exaggerated wound-healing response. Both DD and wound granulation tissue display an increased ratio of type III to type I collagen early on and a corresponding decrease in this collagen ratio in the later stages. ${ }^{39}$ Transforming growth factor (TGF)- $\beta$, a pleotrophic cytokine released during the inflammatory response of wound healing, is highly upregulated in DD. ${ }^{52}$ The cytokine TGF- $\beta 1$ has been shown to promote both fibroblast proliferation through $\beta$-catenin-mediated transactivation of transcription ${ }^{53}$ and myofibroblast differentiation through induction of $\alpha$-smooth muscle actin expression. ${ }^{54}$ We have previously shown that $\beta$-catenin is abundantly present throughout the disease cord, but it is virtually absent from patient-matched phenotypically normal palmar fascia. ${ }^{55}$ Moreover, we have found that when disease cells are cultured on type I collagen in order to more closely recapitulate in vivo conditions, TGF- $\beta 1$ induces $\beta$-catenin 

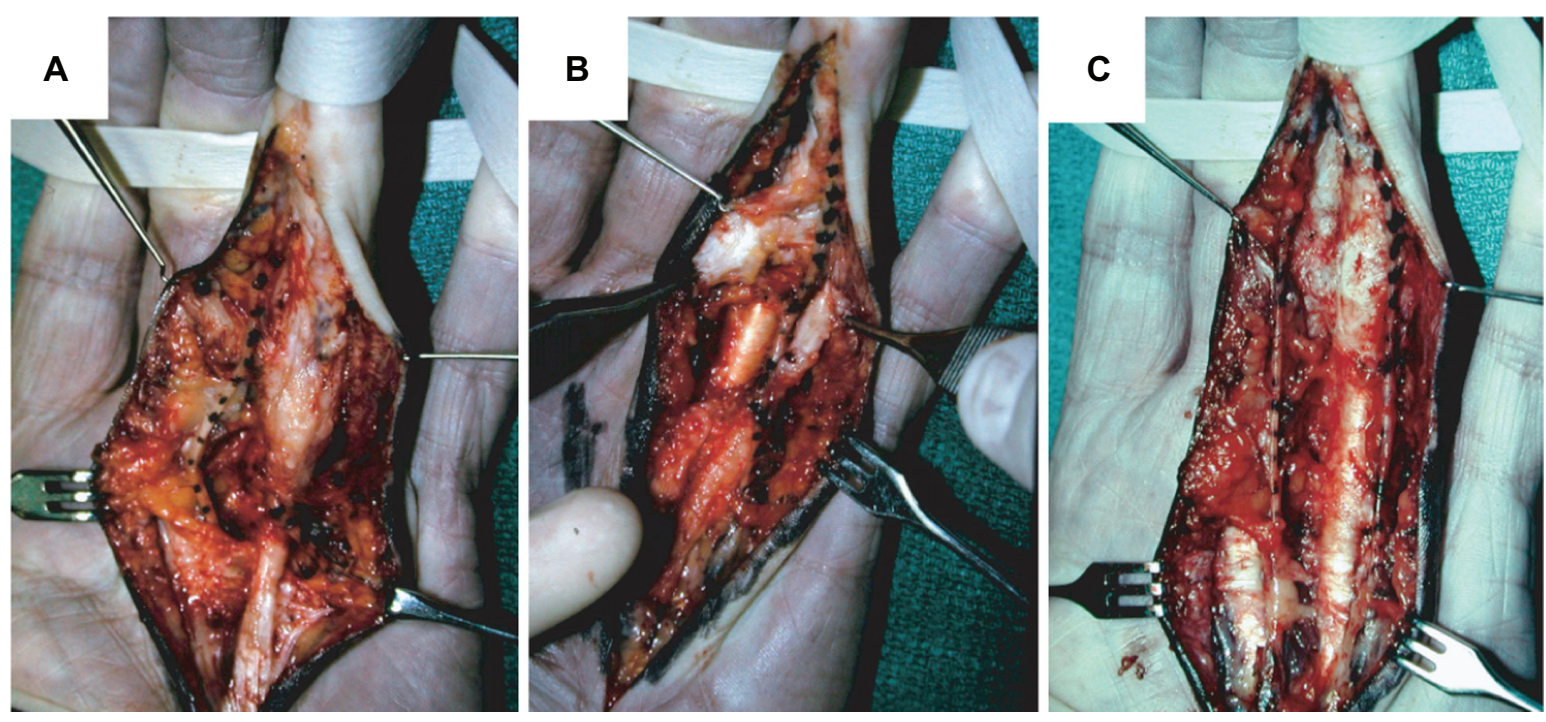

Figure I A and B) The digital neurovascular bundles (stippled) displaced by a spiral cord. C) The digit after resection of the cord, the preserved neurovascular bundle is restored to its normal anatomical position.

accumulation while attenuating levels of $\alpha$-smooth muscle actin. ${ }^{56}$ When disease cells are cultured in stressed 3 -dimensional collagen lattices, TGF- $\beta 1$ stimulation of disease cells showed enhanced cell contractility, correlated with increases in $\alpha$-smooth muscle actin levels and myofibroblast differentiation. ${ }^{57} \mathrm{In}$ addition to TGF- $\beta 1, \mathrm{DD}$ is also characterized by the upregulation of platelet-derived growth factor, basic fibroblast growth factor, and epidermal growth factor, ie, biologically active molecules that are implicated in the various stages of wound healing. ${ }^{58,59}$ These parallels to wound healing in DD have led to the hypothesis that DD may be a response to injury, possibly due to microtrauma or hypoxia, although the evidence in the literature is still inconclusive.

\section{Current and emerging treatments}

Although the current research focus is on the identification of potential molecular therapies for DD management, the most widely practiced intervention continues to be surgical treatment. ${ }^{60}$ Here, we organize current and emerging treatments for DD into three categories of interventions: (1) surgical interventions, (2) physical interventions, and (3) biological interventions.

\section{Surgical interventions}

Surgical intervention, the most commonly used treatment for $\mathrm{DD}$, is typically considered when the contracture significantly affects hand function. This occurs usually when the MCP and/or PIP flexion contracture exceeds $30^{\circ}$. Many techniques are available for addressing finger contractures and joint deformities associated with this condition. One common approach is palmar fasciectomy, which involves the resection of the affected palmar fascia. There are three main variants of palmar fasciectomy: local, regional, and total fasciectomy. Of the three variants, local fasciectomy is the least invasive, which involves the resection of segments of the disease cord, thereby releasing finger contractures ${ }^{61}$ As residual diseased tissue remains in the hand following the local fasciectomy procedure, the chance of recurrence is high ${ }^{61}$ The more common approach is the use of regional or partial fasciectomy, a technique involving the removal of as much of the affected fascia as possible. ${ }^{62}$ This technique is associated with a lower probability of disease recurrence relative to the local fasciectomy procedure ${ }^{61}$ Total or radical fasciectomy is a more invasive procedure, involving the excision of the palmar and digital fascia. ${ }^{61}$ However, this technique is often associated with increased risks of surgical complications and does not have lower recurrence rates when compared to partial fasciectomy. ${ }^{61}$ For less-advanced disease involving only palmar cords, the use of fasciotomy has been advocated. Fasciotomy is a technique used to release contractures by dividing the disease cord without the excision of the diseased tissue. ${ }^{63}$ As the diseased tissue is not removed, the risk for disease recurrence is relatively high. ${ }^{64}$ The closed procedure, often referred to as needle fasciotomy or needle aponeurotomy, involves the placement of a needle adjacent to the cord and the "slicing" of the disease cord with the sharp bevelled edge of the needle to release the contracture. ${ }^{65,66}$ Although this procedure is minimally invasive in nature, many surgeons still believe that it should 
be used with caution as it puts the flexor tendons and nerves at risk for being severed during surgery. ${ }^{66}$ This procedure is currently believed to have a role only in the management of mild-to-moderate MCP contractures. An alternative approach to closed fasciotomy is the open fasciotomy procedure. This approach involves creating an incision in the palm of the hand, allowing for the visualization of the disease cord.$^{67}$ The cord can then be severed, and the contracture is thereby released. ${ }^{67}$ This procedure is less invasive like the closed fasciotomy, but has the advantage that it reduces the risk of damage to the nerves in the hand as it allows for direct visualization of the disease cord.

For the management of aggressive or recurrent disease, dermofasciectomy has been recommended. This extensive technique involves the excision of the diseased fascia en bloc with the overlaying skin, and the subsequent use of skin grafts to close the skin. ${ }^{68,69}$ When compared to fasciectomy alone, dermofasciectomy offers a lower chance of disease recurrence. ${ }^{68}$ Therefore, despite the need for longer postoperative rehabilitation, this technique is sometimes recommended for younger patients who have a history of aggressive disease recurrence.

\section{Physical interventions}

Often in an attempt to improve long-term extension, splinting is prescribed as a postoperative physical therapeutic modality. ${ }^{70}$ Although its effectiveness is still unclear, splinting is used to maximize finger extension and function by promoting wound healing and reducing scar tissue formation. ${ }^{71}$ However, splinting on its own as an alternative to surgery is unlikely to be effective in slowing disease progression or reducing finger contractures or both.

Radiation therapy has also been proposed as an alternative to surgery in early disease stages. ${ }^{72}$ Some clinical studies suggest that radiotherapy may be effective in slowing disease progression. ${ }^{72,73}$ Nonetheless, the effectiveness of radiation therapy has not been convincingly shown, and further work needs to be done to investigate the potential toxicity and long-term risks associated with the use of localized radiation for the treatment of a benign condition.

Another physical modality that has been described in the literature is the use of ultrasound heat therapy. ${ }^{74}$ The regular use of ultrasound can break disulfide bonds that hold collagen fibrils together. When ultrasound is combined with physical stretching, this may lead to improvements in function and may also slow down disease progression. ${ }^{74}$ However, prospective randomized studies have never been performed, and the short- and long-term effectiveness of this treatment modality remains unproved.

\section{Biological interventions}

In light of the high rates of disease recurrence and risks inherent to surgical interventions, the primary focus of most research in DD has been on identifying potential molecular targets for nonsurgical alternative therapies. Differential gene expression analysis combined with molecular and functional studies of diseased tissues and cells have helped identify a number of candidates. One novel treatment modality that has emerged over the years is the use of localized collagenase injections. ${ }^{75-77}$ Since the established disease cords are primarily composed of type I collagen, ${ }^{18,78}$ this makes them susceptible to the enzymatic activity of collagenase, which specifically degrades peptide bonds in collagen fibers. A number of studies have suggested that collagenase injection into disease cords can decrease finger contracture. ${ }^{18,75-77}$ A recent, double-blind, randomized prospective study showed that collagenase injection is an effective nonoperative treatment to reduce contractures and improve joint mobility in advanced disease cases. ${ }^{75}$ Although collagenase injection may reduce some contractures associated with the condition, the long-term rate of recurrence is yet to be determined. One long-term study of collagenase injection for the treatment of DD showed that while the majority of patients treated with collagenase showed reduced joint contractures, of the patients who completed the 8-year follow-up, six had experienced disease recurrence. ${ }^{79}$ In their current form, collagenase injections are, at best, a convenient treatment since the cellular source of the collagen is not targeted by this approach. Finally, since collagenase is not specific to the disease cord structure, the long-term safety of injecting this enzyme into regions of the hand that also contain normal palmar fascia and tendons, which are also composed of type I collagen, is yet to be determined.

The use of corticosteroid injections has been advocated as a treatment for early disease.$^{80}$ It has been used in the clinical setting, and at least in one report, that the intralesional injection of a corticosteroid directly into palmar nodules resulted in the softening and flattening of the disease structures. ${ }^{80}$ This correlates with molecular studies that show that steroids can function as an antifibrotic agent, reducing cell proliferation, while inducing apoptosis or programmed cell death. ${ }^{81,82}$ However, the potential side effects of steroid use, and particularly long-term steroid use, should be considered before treatment. Steroid injections as an antifibrotic treatment have been 
shown to increase patients' risks of skin depigmentation, as well as dermal atrophy from collagen degradation. ${ }^{83,84}$

Other biological approaches that have been tried in clinical settings include the use of enzymatic fasciotomy and $\delta$-interferon injections. In the year 1971, Hueston ${ }^{85}$ suggested that enzymatic fasciotomy can achieve similar outcomes to surgical fasciotomy and may be an appropriate alternative for select patients. Enzymatic fasciotomy can be described as the injection of a cocktail of proteolytic and anti-inflammatory enzymes, targeting the fibroproliferative and collagenous nature of the disease environment. ${ }^{85}$ This injection is accompanied by the physical manipulation and straightening of the digit to break the disease cord and release the contracture. In the year 1992, a study that examined the long-term effects of enzymatic fasciotomy was published. ${ }^{86}$ In this study, although the technique was shown to reduce contracture and improve hand function, there was a rapid rate of early recurrence within 2-3 years among the population studied.$^{86}$ Also, the necessity of forced contracture extension to break the disease cord may result in damage to other parts of the palmar fascia or tendons.

The use of intralesional $\delta$-interferon injections has been described in the literature as a possible treatment for DD. ${ }^{87}$ Molecular studies on cells derived from DD patients indicate that $\delta$-interferon is a cytokine that can reduce cell proliferation, collagen production, and the expression of $\alpha$-smooth muscle actin. ${ }^{88,89}$ Although there are a number of studies showing the potential benefits of using $\delta$-interferon in the treatment of DD, most of these studies have been conducted in vitro and whether these can translate to improvements in a patient is largely unknown. One small pilot study found that injections of $\delta$-interferon led to a decrease in the size of the lesion(s) associated with DD, and that at least in hypertrophic scar tissue, treatment with $\delta$-interferon resulted in a decrease in $\alpha$-smooth muscle actin expression and myofibroblast formation by immunohistochemistry. ${ }^{87}$ This study was not specific to DD and included only four patients with DD in the hand, whereas the remainder presented hypertrophic scars or plantar fibromatosis. Larger and more specific studies on DD patients are required to determine whether the therapeutic benefits of $\delta$-interferon in cell cultures in laboratories can translate to improvements in hand functions in clinical settings.

Other biological agents that may offer therapeutic benefits include calcium channel blockers and matrix metalloproteinase (MMP) inhibitors. Calcium channel blockers, nifedipine and verapamil, have been shown in vitro to inhibit lysophosphatidic acid-induced contraction of DD cells; ${ }^{90}$ but the effects of these blockers on cells derived from normal palmar fascia were not explored. The use of MMP inhibitors has also been proposed in the literature. ${ }^{91-93}$ The MMPs and their inhibitors regulate ECM turnover and function. Studies have found correlations in MMP expression and DD progression and recurrence. ${ }^{93}$ Moreover, it has been shown that the use of ilomastat, a broad MMP inhibitor, decreases mechanical tension generated by disease nodule and cord cells. ${ }^{91}$ Although both calcium channel blockers and MMP inhibitors have been shown to affect molecular processes implicated in the progression of DD, further studies are required to determine the effectiveness of such treatments in improving hand and joint functions in patients.

\section{Discussion and implications for future work}

There is general consensus that a greater understanding of the molecular mechanism of disease progression will lead to the creation of more specific and effective treatment alternatives. Since surgeons do not generally see patients with DD until they are presented with finger contractures, the study of disease progression can be difficult. Moreover, since the treatment of choice in the early stages of DD is observation, the genetic and molecular study of early stage DD is not always feasible. Together, these factors have made the study of disease recurrence an attractive option.

Clinically, the reduction in disease recurrence associated with dermofasciectomy, ${ }^{68}$ which typically removes adjacent tissue in addition to the disease cord, has been interpreted by some to indicate that ECM-cellular interactions promote DD progression and recurrence. ${ }^{94}$ Many of the dysregulated genes that have been identified in DD encode secreted ECM proteins that would be predicted to accumulate in the surrounding fascia. ${ }^{95-99}$ If any of this modified ECM is left behind after the surgical resection of the DD cord, there is the potential for these secreted factors to activate fibroblasts resident in the adjacent fascia to differentiate into myofibroblasts and promote disease recurrence. ${ }^{56}$ However, many molecular studies of DD have elected to study the pathophysiology of these cells using traditional tissue-culture procedures, which removes disease cells from their native environment, negating potential interaction of disease cells with their surrounding microenvironment. Our laboratory has established that culturing of both disease and phenotypically normal fascia cells on type I collagen, which is abundantly found in the disease cord, causes cells to elicit differential responses to TGF- $\beta 1$ treatment, suggesting that 
cell-to-matrix interaction is an important component of the pathology of DD. ${ }^{56}$

While structural components of the ECM such as collagens, laminin, fibronectin, and elastin are often altered in $\mathrm{DD},{ }^{49,100}$ proteases including a disintegrin and metalloprotease domain containing protein 12, MMP-2 and MMP-9, proteoglycans (notably PRG4), and "matricellular" components including tenascin $\mathrm{C}$ and periostin have also been identified as being altered. ${ }^{96,97,99}$ Recently, we have identified that periostin induces proliferation and apoptosis of phenotypically normal fibroblasts derived from the palmar fascia adjacent to the DD cord, while inducing myofibroblast differentiation of DD cells. ${ }^{99}$ ECM molecules such as periostin, which initiate differential response in DD cells and adjacent fibroblasts, may have different roles in DD progression and recurrence and represent attractive targets for therapeutic intervention. The characterization of these proteins, therefore, may identify novel therapeutic targets to prevent DD recurrence.

\section{Conclusion}

In summary, while the use of collagenase is an emerging treatment for the management of DD, it is not curative and the long-term effects are still unknown. For the time being, surgical resection remains the treatment of choice despite the high rate of recurrence associated with this procedure. The recent advances in our understanding of the molecular pathophysiology of this disease have led to the identification of novel molecular targets that hopefully will lead to the creation of more effective treatment alternatives.

\section{Acknowledgments}

We gratefully acknowledge the support of the Institute of Musculoskeletal Health and Arthritis, Canadian Institutes of Health Research (CIHR) summer studentships for Linda Vi. Work in the senior authors' laboratory has been supported by the CIHR, the Canadian and American Societies for Surgery of the Hand, the US Plastic Surgery Educational Foundation, the Lawson Pooled Research Fund, and the University of Western Ontario Department of Surgery.

\section{Disclosure}

The authors declare that they have no competing interests.

\section{References}

1. McFarlane R. Dupuytren's disease. J Hand Ther. 1997;10(1):8-13.

2. de Palma L, Santucci A, Gigante A, Di Giulio A, Carloni S. Plantar fibromatosis: an immunohistochemical and ultrastructural study. Foot Ankle Int. 1999;20(4):253-257.
3. Pratt AL, Byrne G. The lived experience of Dupuytren's disease of the hand. J Clin Nurs. 2009;18(12):1793-1802.

4. Jabaley ME. Surgical treatment of Dupuytren's disease. Hand Clin. 1999;15(1):109-126, vii.

5. Herweijer H, Dijkstra PU, Nicolai JP, van der Sluis CK. Postoperative hand therapy in Dupuytren's disease. Disabil Rehabil. 2007; 29(22):1736-1741.

6. Hindocha S, Stanley JK, Watson S, Bayat A. Dupuytren's diathesis revisited: evaluation of prognostic indicators for risk of disease recurrence. J Hand Surg Am. 2006;31(10):1626-1634.

7. Ross DC. Epidemiology of Dupuytren's disease. Hand Clin. 1999;15(1): 53-62, vi.

8. Childs SG. Dupuytren's disease. Orthop Nurs. 2005;24(2):160-163; quiz 164,165 .

9. Rhomberg M, Rainer C, Gardetto A, Piza-Katzer H. Dupuytren's disease in children-differential diagnosis. J Pediatr Surg. 2002;37(4):E7.

10. Mandalia VI, Lowdon IM. Dupuytren's disease in a child: a case report. J Pediatr Orthop B. 2003;12(3):198-199.

11. Bebbington A, Savage R. Dupuytren's disease in an infant. J Bone Joint Surg Br. 2005;87(1):111-113.

12. Urban M, Feldberg L, Janssen A, Elliot D. Dupuytren's disease in children. J Hand Surg Br. 1996;21(1):112-116.

13. Hueston J. The Dupuytren's diathesis. In: Hueston J, ed. Dupuytren's Contracture. Edinburgh, UK: E \& S Livingstone; 1963:51-63.

14. McFarlane RM. On the origin and spread of Dupuytren's disease. J Hand Surg Am. 2002;27(3):385-390.

15. Egawa T, Senrui H, Horiki A, Egawa M. Epidemiology of the Oriental patient. In: McFarlane RM, McGrouther DA, Flint MH, eds. Dupuytren's Disease Biology and Treatment. Edinburgh, UK: Churchill Livingstone; 1990:239-245.

16. Gudmundsson K, Arngrimsson R, Sigfússon N, Björnsson Á, Jónsson T. Epidemiology of Dupuytren's disease: clinical, serological, and social assessment. The Reykjavik Study. J Clin Epidemiol. 2000;53(3):291-296.

17. Cagliero E, Apruzzese W, Perlmutter GS, Nathan DM. Musculoskeletal disorders of the hand and shoulder in patients with diabetes mellitus. Am J Med. 2002;112(6):487-490.

18. Arkkila PE, Koskinen PJ, Kantola IM, Ronnemaa T, Seppanen E, Viikari JS. Dupuytren's disease in type I diabetic subjects: investigation of biochemical markers of type III and I collagen. Clin Exp Rheumatol. 2000;18(2):215-219.

19. Arafa M, Noble J, Royle SG, Trail IA, Allen J. Dupuytren's and epilepsy revisited. J Hand Surg Br. 1992;17(2):221-224.

20. Burge P, Hoy G, Regan P, Milne R. Smoking, alcohol and the risk of Dupuytren's contracture. J Bone Joint Surg Br. 1997;79(2):206-210.

21. Godtfredsen NS, Lucht H, Prescott E, Sorensen TI, Gronbaek M. A prospective study linked both alcohol and tobacco to Dupuytren's disease. J Clin Epidemiol. 2004;57(8):858-863.

22. Gudmundsson KG, Arngrimsson R, Jonsson T. Dupuytren's disease, alcohol consumption and alcoholism. Scand J Prim Health Care. 2001;19(3):186-190.

23. Lucas G, Brichet A, Roquelaure Y, Leclerc A, Descatha A. Dupuytren's disease: personal factors and occupational exposure. Am J Ind Med. 2008;51(1):9-15.

24. Logan AJ, Mason G, Dias J, Makwana N. Can rock climbing lead to Dupuytren's disease? Br J Sports Med. 2005;39(9):639-644.

25. Thomas P, Clarke D. Vibration white finger and Dupuytren's contracture: are they related? Occup Med. 1992;42(3):155.

26. Abe Y, Rokkaku T, Ebata T, Tokunaga S, Yamada T. Dupuytren's disease following acute injury in Japanese patients: Dupuytren's disease or not? J Hand Surg Eur Vol. 2007;32(5):569-572.

27. Liss GM, Stock SR. Can Dupuytren's contracture be work-related?: review of the evidence. Am J Ind Med. 1996;29(5):521-532.

28. Mikkelsen OA. Dupuytren's disease - a study of the pattern of distribution and stage of contracture in the hand. Hand. 1976;8(3): 265-271.

29. Chiu HF, McFarlane RM. Pathogenesis of Dupuytren's contracture: a correlative clinical-pathological study. J Hand Surg Am. 1978;3(1):1-10. 
30. McFarlane R. Patterns of the diseased fascia in the fingers in Dupuytren's contracture. Plast Reconstr Surg. 1974;54(1):31.

31. Rayan GM. Dupuytren disease: anatomy, pathology, presentation, and treatment. J Bone Joint Surg Am. 2007;89(1):189-198.

32. Mikkelsen O. Knuckle pads in Dupuytren's disease. J Hand Surg Eur Vol. 1977;9(3):301-305.

33. Garrod A. On an unusual form of nodule upon the joints of the fingers. St Bartholomews Hosp Rev. 1893;29:157-161.

34. Garrod A. Concerning pads upon the finger joint and their clinical relationship. Br Med J. 1904;2:8.

35. Parker HG. Dupuytren's contracture as a cause of stenosing tenosynovitis. J Maine Med Assoc. 1979;70(4):147-148.

36. Luck JV. Dupuytren's contracture: a new concept of the pathogenesis correlated with surgical management. J Bone Joint Surg Am. 1959; 41-A(4):635-664.

37. Rayan GM. Palmar fascial complex anatomy and pathology in Dupuytren's disease. Hand Clin. 1999;15(1):73-86, vi-vii.

38. Watson H, Paul H Jr. Pathologic anatomy. Hand clin. 1991;7(4):661.

39. Lam WL, Rawlins JM, Karoo RO, Naylor I, Sharpe DT. Re-visiting Luck's classification: a histological analysis of Dupuytren's disease. J Hand Surg Eur Vol. 2010;35(4):312-317.

40. Murrell GA, Francis MJ, Howlett CR. Dupuytren's contracture. Fine structure in relation to aetiology. J Bone Joint Surg Br. 1989;71(3): 367-373.

41. Murrell GA, Hueston JT. Aetiology of Dupuytren's contracture. Aust N Z J Surg. 1990;60(4):247-252.

42. Wilutzky B, Berndt A, Katenkamp D, Koshmehl H. Programmed cell death in nodular palmar fibromatosis (Morbus Dupuytren). Histol Histopathol. 1998;13(1):67-72.

43. Verjee LS, Midwood K, Davidson D, Essex D, Sandison A, Nanchahal J. Myofibroblast distribution in Dupuytren's cords: correlation with digital contracture. J Hand Surg Am. 2009;34(10):1785-1794.

44. Tomasek J, Rayan GM. Correlation of alpha-smooth muscle actin expression and contraction in Dupuytren's disease fibroblasts. J Hand Surg Am. 1995;20(3):450-455.

45. Bisson MA, McGrouther DA, Mudera V, Grobbelaar AO. The different characteristics of Dupuytren's disease fibroblasts derived from either nodule or cord: expression of alpha-smooth muscle actin and the response to stimulation by TGF-beta1. J Hand Surg Br. 2003;28(4):351-356.

46. Perez-Montiel MD, Plaza JA, Dominguez-Malagon H, Suster S. Differential expression of smooth muscle myosin, smooth muscle actin, h-caldesmon, and calponin in the diagnosis of myofibroblastic and smooth muscle lesions of skin and soft tissue. Am J Dermatopathol. 2006;28(2):105-111.

47. Welch MP, Odland GF, Clark RA. Temporal relationships of F-actin bundle formation, collagen and fibronectin matrix assembly, and fibronectin receptor expression to wound contraction. J Cell Biol. 1990;110(1):133-145

48. Hinz B. Masters and servants of the force: the role of matrix adhesions in myofibroblast force perception and transmission. Eur J Cell Biol. 2006;85(3-4):175-181.

49. Howard JC, Varallo VM, Ross DC, et al. Wound healing-associated proteins Hsp47 and fibronectin are elevated in Dupuytren's contracture. J Surg Res. 2004;117(2):232-238.

50. Magro G, Lanzafame S, Micali G. Co-ordinate expression of alpha 5 beta 1 integrin and fibronectin in Dupuytren's disease. Acta Histochem. 1995;97(3):229-233.

51. Magro G, Fraggetta F, Travali S, Lanzafame S. Immunohistochemical expression and distribution of alpha2beta1, alpha6beta1, alpha5beta1 integrins and their extracellular ligands, type IV collagen, laminin and fibronectin in palmar fibromatosis. Gen Diagn Pathol. 1997;143(4):203-208

52. Badalamente MA, Sampson SP, Hurst LC, Dowd A, Miyasaka K. The role of transforming growth factor beta in Dupuytren's disease. J Hand Surg Am. 1996;21(2):210-215.
53. Cheon SS, Nadesan P, Poon R, Alman BA. Growth factors regulate beta-catenin-mediated TCF-dependent transcriptional activation in fibroblasts during the proliferative phase of wound healing. Exp Cell Res. 2004;293(2):267-274.

54. Serini G, Gabbiana G. Modulation of alpha-smooth muscle actin expression in fibroblasts by transforming growth factor-beta isoforms: an in vivo and in vitro study. Wound Repair Regen. 1996;4(2) 278-287.

55. Varallo VM, Gan BS, Seney S, et al. Beta-catenin expression in Dupuytren's disease: potential role for cell-matrix interactions in modulating beta-catenin levels in vivo and in vitro. Oncogene. 2003; 22(24):3680-3684.

56. Vi L, Njarlangattil A, Wu Y, Gan BS, O'Gorman DB. Type-1 collagen differentially alters beta-catenin accumulation in primary Dupuytren's disease cord and adjacent palmar fascia cells. BMC Musculoskelet Disord. 2009;10:72.

57. Bisson M, Beckett K, McGrouther D, Grobbelaar A, Mudera V. Transforming growth factor-[beta] 1 stimulation enhances Dupuytren's fibroblast contraction in response to uniaxial mechanical load within a 3-dimensional collagen gel. J Hand Surg Am. 2009;34(6): $1102-1110$.

58. Alioto RJ, Rosier RN, Burton RI, Puzas JE. Comparative effects of growth factors on fibroblasts of Dupuytren's tissue and normal palmar fascia. J Hand Surg Am. 1994;19(3):442-452.

59. Augoff K, Kula J, Gosk J, Rutowski R. Epidermal growth factor in Dupuytren's disease. Plast Reconstr Surg. 2005;115(1):128-133.

60. Elliot D. The early history of Dupuytren's disease. Hand Clin. 1999; 15(1): 1-19, v.

61. Zachariae L. Extensive versus limited fasciectomy for Dupuytren's contracture. Scand J Plast Reconstr Surg Hand Surg. 1967;1(2): $150-153$.

62. Coert J, Nérin J, Meek M. Results of partial fasciectomy for Dupuytren disease in 261 consecutive patients. Ann Plast Surg. 2006;57(1):13.

63. Rowley DI, Couch M, Chesney RB, Norris SH. Assessment of percutaneous fasciotomy in the management of Dupuytren's contracture. $J$ Hand Surg Br. 1984;9(2):163-164.

64. van Rijssen A, Gerbrandy F, Linden H, Klip H, Werker P. A comparison of the direct outcomes of percutaneous needle fasciotomy and limited fasciectomy for Dupuytren's disease: a 6-week follow-up study. J Hand Surg Am. 2006;31(5):717-725.

65. Cheng H, Hung L, Tse W, Ho P. Needle aponeurotomy for Dupuytren's contracture. J Orthop Surg. 2008;16(1):88-90.

66. Foucher G, Medina J, Navarro R. Percutaneous needle aponeurotomy: complications and results. J Hand Surg Br. 2003;28(5):427-431.

67. Jabecki J, Kaczmarzyk L, Domanasiewicz A, Kaczmarzyk J. Early results of open fasciotomy as a treatment of Dupuytren's disease in elderly patients. Pol J Surg. 2007;79(12):746-752.

68. Brotherston T, Balakrishnan C, Milner R, Brown H. Long term follow-up of dermofasciectomy for Dupuytren's contracture. Br J Plast Surg. 1994;47(6):440-443.

69. Armstrong J, Hurren J, Logan A. Dermofasciectomy in the management of Dupuytren's disease. J Bone Joint Surg Br. 2000;82(1):90-94.

70. Larson D, Jerosch-Herold C. Clinical effectiveness of post-operative splinting after surgical release of Dupuytren's contracture: a systematic review. BMC Musculoskelet Disord. 2008;9:104.

71. Evans RB, Dell PC, Fiolkowski P. A clinical report of the effect of mechanical stress on functional results after fasciectomy for Dupuytren's contracture. $J$ Hand Ther. 2002;15(4):331-339.

72. Seegenschmiedt MH, Olschewski T, Guntrum F. Radiotherapy optimization in early-stage Dupuytren's contracture: first results of a randomized clinical study. Int J Radiat Oncol Biol Phys. 2001;49(3):785-798.

73. Keilholz L, Seegenschmiedt MH, Sauer R. Radiotherapy for prevention of disease progression in early-stage Dupuytren's contracture: initial and long-term results. Int J Radiat Oncol Biol Phys. 1996;36(4):891-897.

74. Stiles PJ. Ultrasonic therapy in Dupuytren's contracture. J Bone Joint Surg Br. 1966;48(3):452-454. 
75. Hurst LC, Badalamente MA, Hentz VR, et al. Injectable collagenase clostridium histolyticum for Dupuytren's contracture. $N$ Engl J Med. 2009;361(10):968-979.

76. Badalamente MA, Hurst LC, Hentz VR. Collagen as a clinical target: nonoperative treatment of Dupuytren's disease. J Hand Surg Am. 2002;27(5):788-798.

77. Badalamente MA, Hurst LC. Efficacy and safety of injectable mixed collagenase subtypes in the treatment of Dupuytren's contracture. J Hand Surg Am. 2007;32(6):767-774.

78. Bailey AJ, Sims TJ, Gabbiani G, Bazin S, LeLous M. Collagen of Dupuytren's disease. Clin Sci Mol Med. 1977;53(5):499-502.

79. Watt AJ, Curtin CM, Hentz VR. Collagenase injection as nonsurgical treatment of Dupuytren's disease: 8-year follow-up. J Hand Surg Am. 2010;35(4):534-539,539.e1.

80. Ketchum LD, Donahue TK. The injection of nodules of Dupuytren's disease with triamcinolone acetonide. J Hand Surg Am. 2000;25(6): 1157-1162.

81. Pagnotta A, Specchia N, Soccetti A, Manzotti S, Greco F. Responsiveness of Dupuytren's disease fibroblasts to 5 alpha-dihydrotestosterone. J Hand Surg Am. 2003;28(6):1029-1034.

82. Meek RM, McLellan S, Reilly J, Crossan JF. The effect of steroids on Dupuytren's disease: role of programmed cell death. J Hand Surg Br. 2002;27(3):270-273.

83. Newman R. Local skin depigmentation due to corticosteroid injection Br Med J (Clin Res Ed). 1984;288(6432):1725-1726.

84. Oikarinen A, Autio P. New aspects of the mechanism of corticosteroidinduced dermal atrophy. Clin Exp Dermatol. 1991;16(6):416-419.

85. Hueston JT. Enzymic fasciotomy. Hand. 1971;3(1):38-40.

86. McCarthy DM. The long-term results of enzymic fasciotomy. J Hand Surg Br. 1992;17(3):356.

87. Pittet B, Rubbia-Brandt L, Desmouliere A, et al. Effect of gammainterferon on the clinical and biologic evolution of hypertrophic scars and Dupuytren's disease: an open pilot study. Plast Reconstr Surg. 1994;93(6):1224-1235.

88. Sanders JL, Dodd C, Ghahary A, Scott PG, Tredget EE. The effect of interferon-alpha2b on an in vitro model Dupuytren's contracture. J Hand Surg Am. 1999;24(3):578-585.

89. Tanaka K, Sano K, Nakano T, Yuba K, Kinoshita M. Suppression of alpha smooth muscle actin expression by IFN-gamma in established myofibroblast cell lines. J Interferon Cytokine Res. 2007;27(10):835-839.
90. Rayan GM, Parizi M, Tomasek JJ. Pharmacologic regulation of Dupuytren's fibroblast contraction in vitro. J Hand Surg Am. 1996;21(6):1065-1070.

91. Townley WA, Cambrey AD, Khaw PT, Grobbelaar AO. The role of an MMP inhibitor in the regulation of mechanical tension by Dupuytren's disease fibroblasts. J Hand Surg Eur. 2009;34(6):783-787.

92. Townley WA, Cambrey AD, Khaw PT, Grobbelaar AO. Matrix metalloproteinase inhibition reduces contraction by dupuytren fibroblasts. J Hand Surg Am. 2008;33(9):1608-1616.

93. Johnston P, Larson D, Clark IM, Chojnowski AJ. Metalloproteinase gene expression correlates with clinical outcome in Dupuytren's disease. J Hand Surg Am. 2008;33(7):1160-1167.

94. Vi L, Gan BS, O'Gorman DB. The potential roles of cell migration and extra-cellular matrix interactions in Dupuytren's disease progression and recurrence. Med Hypotheses. 2010;74(3):510-512.

95. Qian A, Meals RA, Rajfer J, Gonzalez-Cadavid NF. Comparison of gene expression profiles between Peyronie's disease and Dupuytren's contracture. Urology. 2004;64(2):399-404.

96. Rehman S, Salway F, Stanley JK, Ollier WE, Day P, Bayat A. Molecular phenotypic descriptors of Dupuytren's disease defined using informatics analysis of the transcriptome. J Hand Surg Am. 2008;33(3):359-372.

97. Shih B, Wijeratne D, Armstrong DJ, Lindau T, Day P, Bayat A. Identification of biomarkers in Dupuytren's disease by comparative analysis of fibroblasts versus tissue biopsies in disease-specific phenotypes. J Hand Surg Am. 2009;34(1):124-136.

98. Satish L, Laframboise WA, O'Gorman DB, et al. Identification of differentially expressed genes in fibroblasts derived from patients with Dupuytren's contracture. BMC Med Genomics. 2008;1:10

99. Vi L, Feng L, Zhu RD, et al. Periostin differentially induces proliferation, contraction and apoptosis of primary Dupuytren's disease and adjacent palmar fascia cells. Exp Cell Res. 2009;315(20):3574-3586.

100. Howard JC, Varallo VM, Ross DC, et al. Elevated levels of beta-catenin and fibronectin in three-dimensional collagen cultures of Dupuytren's disease cells are regulated by tension in vitro. BMC Musculoskelet Disord. 2003;4:16.
Orthopedic Research and Reviews

\section{Publish your work in this journal}

Orthopedic Research and Reviews is an international, peer-reviewed, open access journal focusing on the patho-physiology of the musculoskeletal system, trauma, surgery and other corrective interventions to restore mobility and function. Advances in new technologies, materials, techniques and pharmacological agents are particularly welcome. The journal welcomes

\section{Dovepress}

original research, clinical studies, reviews \& evaluations, expert opinion and commentary, case reports and extended reports. The manuscript management system is completely online and includes a very quick and fair peer-review system, which is all easy to use. Visit http://www.dovepress. com/testimonials.php to read real quotes from published authors. 